> Les virus de l'immunodéficience humaine VIH-1 et VIH-2 sont le résultat de plusieurs transmissions inter-espèces de virus simiens à I'homme. II a été montré récemment que le SIVcpzPtt, à l'origine du VIH-l des groupes $M$ et $N$, est toujours présent dans les populations de chimpanzés Pan troglodytes troglodytes du Sud Cameroun. L'ancêtre du VIH-l du groupe 0 est présent chez le gorille de l'Ouest (Gorilla gorilla) mais les chimpanzés sont le réservoir original du SIVgor et il reste à établir si le VIH1 du groupe 0 a été transmis à l'homme par le gorille et/ou le chimpanzé. Au moins huit transmissions inter-espèces de SIVsmm ont eu lieu entre l'homme et le mangabey enfumé, correspondant aux 8 groupes HIV-2. Dans la mesure où l'homme est toujours potentiellement exposé à de nombreux SIV du fait de la chasse ou de la préparation de viande de brousse, la possibilité de nouveaux épisodes de transmissions inter-espèces de lentivirus de primates est une éventualité qu'il ne faut pas exclure. <

\section{Phylogénie des SIV et des VIH}

Mieux comprendre l'origine des VIH

Martine Peeters, Marie-Laure Chaix, Eric Delaporte

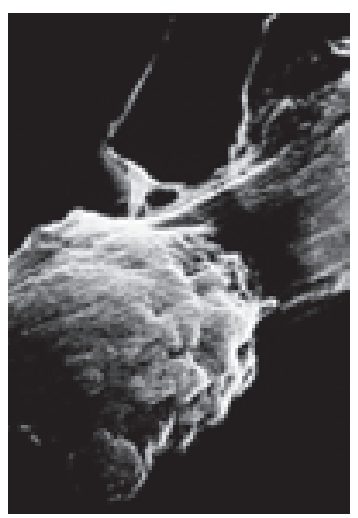

M. Peeters, $\varepsilon$. Delaporte : UMR 145, Institut de Recherche pour le développement (IRD), Université de Montpellier, 34000 Montpellier, France. martine.peeters@mpl.ird.fr M.L. Chaix : EA 3620 MRT, Université Paris Descartes, Laboratoire de Virologie, CHU Necker-Enfants Malades, 149, rue de Sèvres, 75015 Paris, France. marie-laure.chaix@nck.aphp.fr

\section{Taxonomie, classification} et répartition des rétrovirus

Le VIH fait partie de la famille des rétrovirus. Ces virus sont très répandus dans le monde animal. La présence d'une enzyme, la transcriptase inverse, permettant la rétrotranscription de l'ARN en ADN caractérise ces virus. On distingue actuellement trois sous-groupes au sein des rétrovirus humains: les oncovirus à ARN de type HTLV (human T-lymphotropic virus), les spumavirus et enfin les lentivirus dont font partie les $\mathrm{VIH}$. Les $\mathrm{VIH}-1$ et $\mathrm{VIH}-2$, agents étiologiques du Sida chez l'Homme, sont apparentés aux lentivirus de primates appelés SIV pour simian immunodeficiency virus. Les SIV les plus proches du VIH-l sont le SIVcpz et SIVgor qui infectent naturellement les chimpanzés (Pan troglodytes troglodytes) et les gorilles (Gorilla gorilla) d'Afrique Centrale de l'ouest. Les SIVsmm retrouvés chez les mangabeys enfumés (Cercocebus atys) d'Afrique de l'Ouest sont les plus proches pour le VIH-2 [1-3].

Les virus de l'immunodéficience simienne (SIV) Les SIV provenant de différentes espèces de primates sont désignés à l'aide d'un code de trois lettres faisant référence au nom commun en anglais de leur espèce d'origine (par exemple SIVcpz pour chimpanzé). Lorsque différentes sous-espèces d'une 
même espèce de primate sont infectées par un SIV spécifique, le nom de cette sous-espèce est ajouté; c'est le cas pour les SIVcpzPtt et SIVcpzPts qui infectent deux des quatre sous-espèces de chimpanzés, le P. t. troglodytes et le P. t. schweinfurthii.

Actuellement, à la suite d'études sérologiques (recherche d'anticorps anti-SIV), ce sont 39 espèces de primates qui semblent infectées par un SIV. Pour 32 d'entre elles (Tableau I) une confirmation moléculaire a été obtenue, et un génome SIV complet est disponible pour 19 virus. D’une façon générale, une grande diversité génétique est observée au sein des SIV, mais habituellement, chaque espèce de singe est infectée par un SIV qui lui est spécifique [4-6] (Figure 1). Un point majeur est le fait que bien que ces virus se nomment virus de l'immunodéficience simienne, ils n'entraînent pas de Sida chez leur hôte naturel, suggérant une adaptation évolutive depuis une longue période [34]. Il est important de souligner que sur les 69 espèces de primates africains, 30 espèces n’ont jamais été étudiées ce qui laisse suggèrer que de nombreux SIV restent encore à décrire [7]. Fait important à noter, seuls les primates africains sont porteurs de SIV, aucun SIV n'a été identifié chez les primates asiatiques ou du nouveau monde.

\section{Les virus de l'immunodéficience humaine (VIH)}

Les virus $\mathrm{VIH}-\mathrm{l}$ sont actuellement classés en trois groupes: le groupe $M$ (responsable de la pandémie), le groupe 0 (outlier) et le groupe $N$ (non-M non-0). Le groupe $M$ est actuellement subdivisé en 9 sous-types ( $A, B, C, D, F, G, H, J, K)$ et 37 formes recombinantes (CRF0l-CRF37) (http://hiv-web.lanl.gov). Le sous-type $A$ est subdivisé en sous-sous-type $A 1, A 2$ et plus récemment $A 3$ et $A 4$ [8]. Le sous-type $F$ est lui-même subdivisé en sous-sous-type $\mathrm{Fl}$ et $\mathrm{F} 2$.

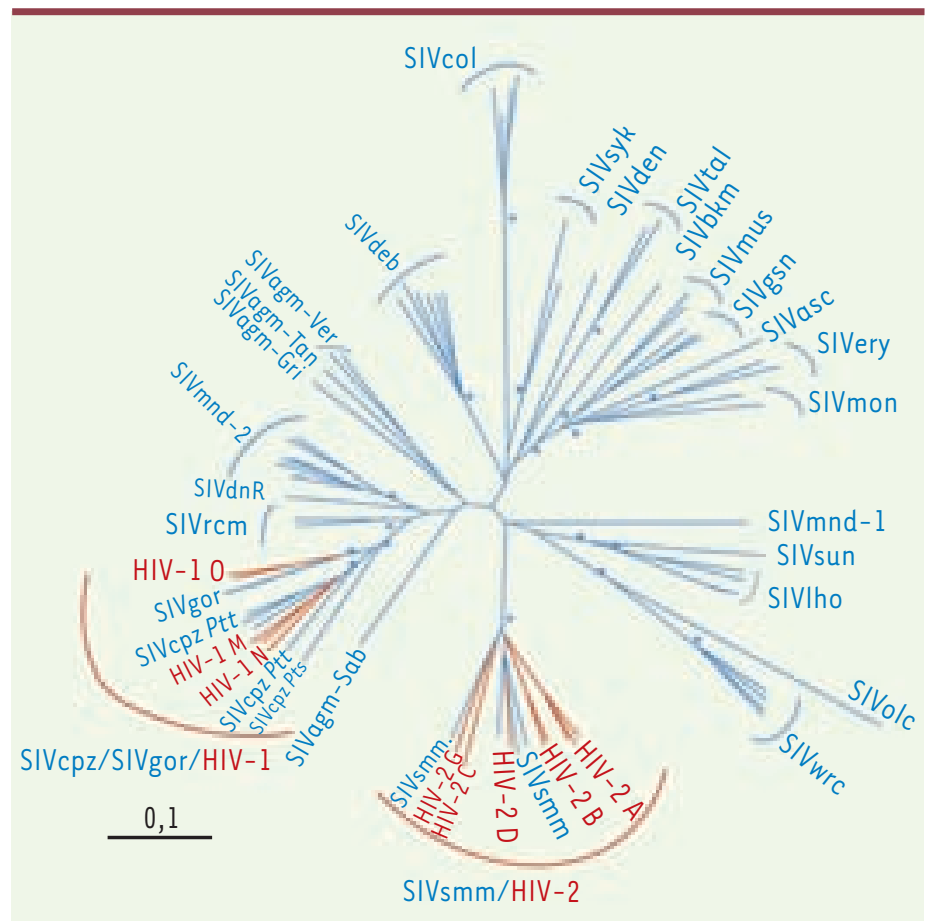

Pour proposer un nouveau sous-type ou une nouvelle forme recombinante (CRF), la nomenclature impose l'identification de 3 virus séquencés sur la totalité du génome et sans lien épidémiologique entre eux. De très nombreuses formes recombinantes ne correspondant pas à ces critères de classification sont appelées URF (unique recombinant forms). Ces formes recombinantes proviennent d'évènements de recombinaison intervenus lors de la rétrotranscription de leurs ARN dans une cellule co-infectée par différents sous-types.

Parmi les CRF, les plus connues et fréquemment retrouvées sont le CRFOl-AE (recombinaison entre les virus de sous-type $A$ et les virus de sous-type $\varepsilon$, virus probablement ancestral mais non décrit à ce jour) responsable d'une partie de l'épidémie en Asie, et le CRF02-AG (recombinaison entre les virus de sous-type $A$ et les virus de sous-type $G$ ) responsable de l'épidémie en Afrique de l'Ouest.

La répartition géographique des différents VIH-1 diffère selon l'ancienneté de l'épidémie dans le pays, l'effet fondateur dans le groupe d'individus considéré et selon les migrations de population (Figure 2). Dans les pays du Nord, le sous-type B est prédominant et il est responsable de l'épidémie initiale dans les pays industrialisés, en particulier l'Amérique du Nord et l'Europe de l'Ouest, dont la France. Par contre à l'échelle mondiale, les $\mathrm{VIH}-1$ non-B représentent au moins $90 \%$ des virus circulants [9]. Les sous-types prédominants sont le sous-type $C$, responsable de $50 \%$ des infections, principalement en Afrique du Sud et en Asie, le sous-type A et le soustype $D$ en Afrique de l' $\varepsilon s t$, le CRF02-AG en Afrique de l'Ouest et le sous-type $B(12 \%)$ en Amérique du Nord et en Europe. En Afrique centrale, circulent les différents groupes du VIH-I (M, N et 0$)$, l'ensemble des sous-types du groupe $M$ et la majorité des formes recombinantes.

En France, on constate une évolution de l'épidémie puisque pour les patients diagnostiqués au moment de leur primo-infection, la fréquence de virus VIH-l de sous-type non-B est passée de $10 \%$ durant les années 1999-2000 à $28 \%$ pour les patients infectés en 2004-2006 [10-12]. Chez les patients nouvelle-

Figure 1. Arbre phylogénétique des différents SIV et VIH fondé sur les séquences du gène pol. L'arbre phylogénétique inclut 26 des 32 SIV infectant des primates non-humains et pour lesquels des séquences pol sont disponibles. Les isolats de HIV résultant de transmissions inter-espèces à partir de SIV sont indiqués en rouge sur l'arbre. Les astérisques $(*)$ correspondent à des valeurs de bootstraps $>80 \%$. 


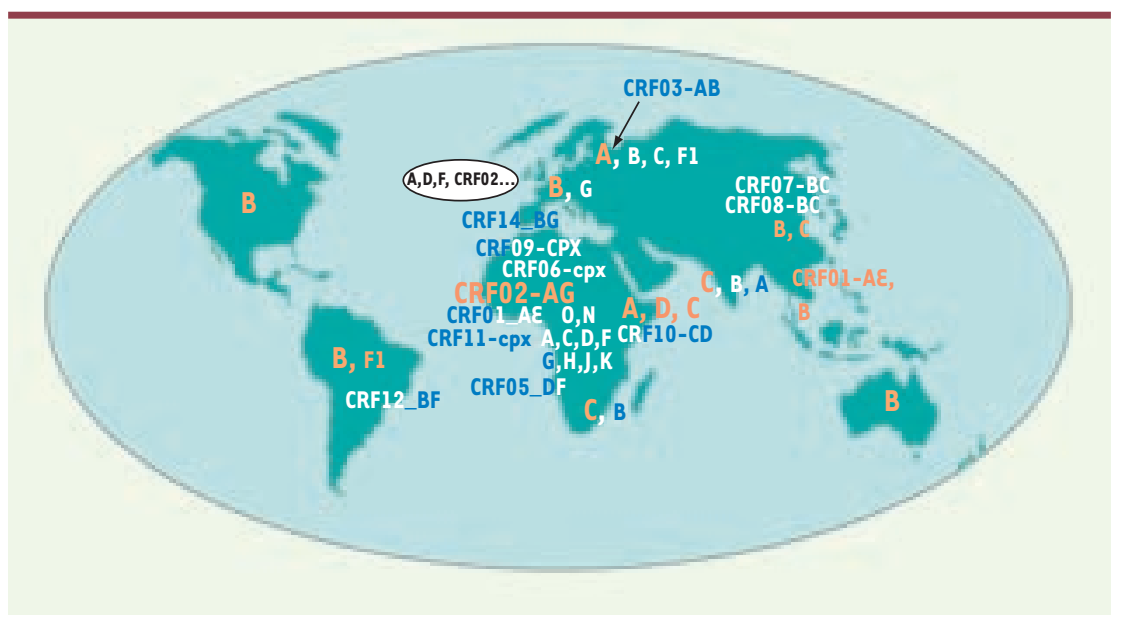

Figure 2. Répartition géographique des différents sous-types et CRF (formes recombinantes circulantes) du VIH-1 groupe M ainsi que des VIH-1 groupe $\mathrm{N}$ et VIH-1 groupe 0 . chimpanzés sont connus pour chasser et se nourrir d'autres primates, tels que les hocheurs (greater spot-nosed guenons -gsn), Cercopithecus nictitans), différentes espèces de colobes... Ainsi la région 5' du génome de SIVcpz (gag, pol, vif et vpr) est très proche de celle du SIVrcm (red capped mangabey, Cercocebus torquatus) alors que la région 3' (vpu, env, et nef) est, quant à elle, très proche de celle du SIVgsn du hocheur. Une recombinaison entre ces 2 virus (SIVgsn et SIVrcm) a probablement eu lieu chez le chimpanzé et ce virus recombinant est à l'origine de l'ancêtre commun de la lignée SIVcpz, lequel fut par la suite transmis à l'homme [14]. ment dépistés en France, quel que soit leur stade clinique, la proportion de ceux qui sont infectés par des virus non-B est de $50 \%$ [13]. Cette augmentation résulte de deux phénomènes: une proportion plus importante de patients originaires d'Afrique sub-saharienne et une augmentation de la fréquence des virus non-B dans la population caucasienne, en raison de la mixité des populations. La moitié des virus non-B isolés en France sont des virus CRF02-AG, ce qui témoigne des liens existant entre la France et l'Afrique de l'Ouest.

À la différence du VIH-1, un nombre limité de souches VIH-2 a été génétiquement caractérisé permettant une classification en 8 groupes $(A-H)$, seuls les groupes $A$ et $B$ jouant un rôle dans l'épidémie du VIH-2 en Afrique de l'Ouest.

\section{Phylogénie des lentivirus de primates}

La diversité génétique parmi les lentivirus de primates (non-humains) est extrêmement complexe. Des exemples de co-évolution entre des virus et leur hôte ont été décrits, mais également des exemples de recombinaison entre des SIV relativement distants, enfin une même espèce de primates peut être porteuse de deux SIV différents. Ces éléments impliquent la survenue relativement fréquente d'épisodes de transmissions inter-espèces et de phénomènes de surinfection. Par la suite, ces SIV peuvent évoluer différemment dans leur nouvel hôte et être à l'origine de nouveaux recombinants pouvant ensuite connaître une diffusion importante [5-7]. Un exemple des plus emblématiques de transmission inter-espèces suivie d'une recombinaison est le SIVcpz du chimpanzé. Les
Les chimpanzés peuvent être divisés en quatre sous-espèces sur la base des séquences de leur ADN mitochondrial (mtADN). Ces quatre sous-espèces ont une répartition géographique précise: le Pan troglodytes verus en Afrique de l'Ouest, le Pan troglodytes troglodytes en Afrique Centrale de l'Ouest, le Pan troglodytes schweinfurthii en Afrique Centrale de l'Est et le Pan troglodytes vellerosus dont la répartition géographique limitée se situe entre la rivière Cross du Nigéria et la rivière Sanaga au Cameroun. Dans la mesure ou aucun SIV n'a été retrouvé chez le P.t.verus et le P.t.vellerosus, I'hypothèse la plus probable est que la transmission inter-espèce de ce virus recombinant ou de son ancêtre a du se passer après la différenciation des sous-espèces verus et vellerosus, mais avant celle des troglodytes et des schweinfurthii toutes deux infectées par un SIVcpz spécifique [15-17].

\section{Origine des VIH}

\section{Origine du VIH-2}

Peu de temps après la découverte en 1983 du VIH-1, le premier SIV, SIVmac, fut isolé du macaque rhésus (Macaca mulatta) dans un centre de primatologie aux États-Unis, le New England Regional Primate Research Center (NERPRC). Des études rétrospectives montrèrent que ce virus fut introduit au NERPRC par d'autres singes rhésus provenant d'un centre de primatologie Californien où s'était développée à la fin des années 1960 une épidémie chez les singes, caractérisée par la survenue d'une immunodépression avec l'apparition d'infections opportunistes. Une même infection fut également décrite chez d'autres macaques (Stump tailed macaques, Macaca arctoides). Dans les deux cas, les macaques infectés avaient été en contact avec des mangabeys enfumés (C.atys), diagnostiqués rétrospectivement comme porteurs du SIVsmm [18]. La relation phylogénétique proche entre SIVmac, SIVstm et SIVsmm suggère que le virus du mangabey enfumé (SIVsmm) était la source de contamination chez les macaques. Ce changement d'hôte avait conduit chez le macaque 


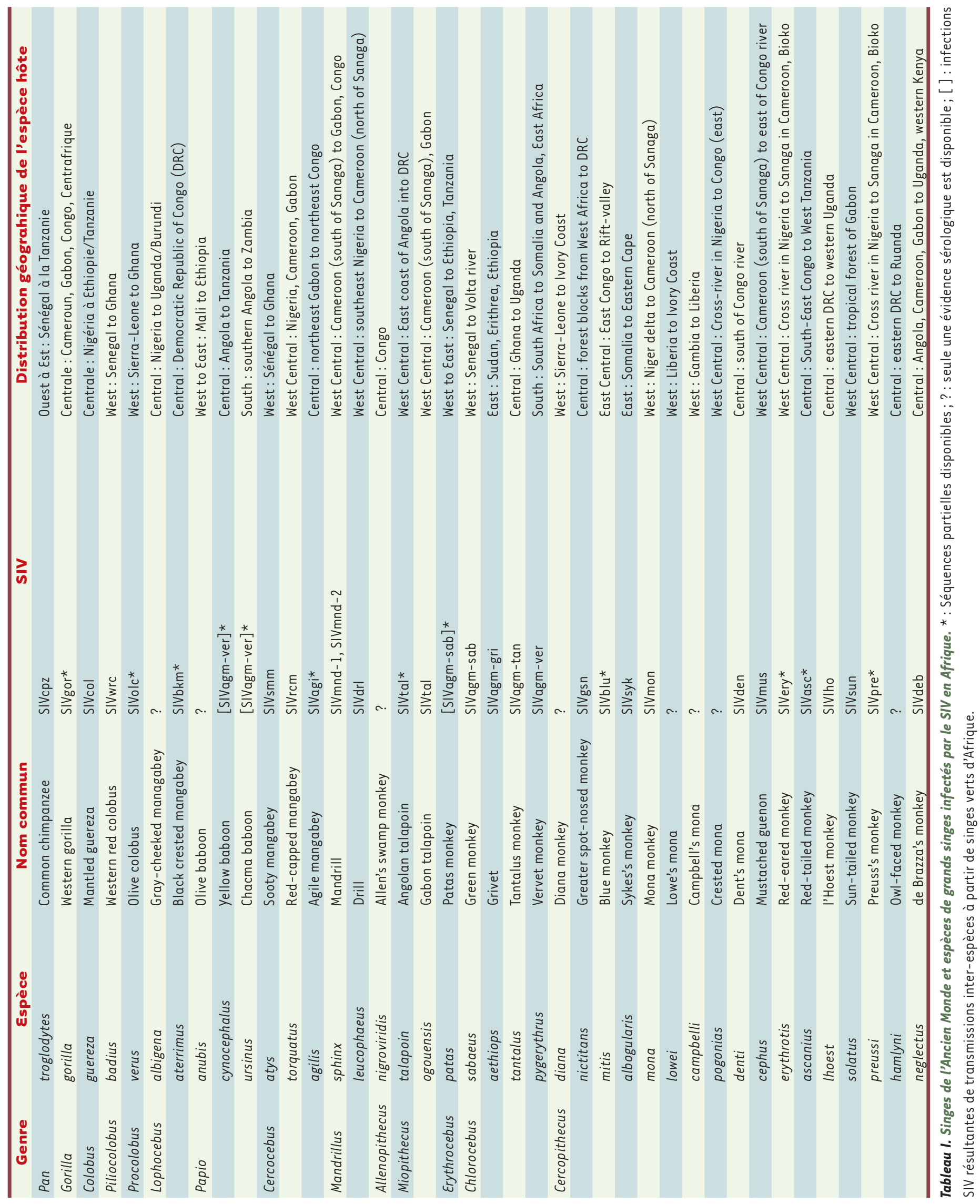


à une maladie remarquablement proche du Sida. Dès lors, I'origine simienne du VIH fut suspectée.

En 1986, la découverte du VIH-2 et sa remarquable homologie avec le SIVsmm infectant naturellement le mangabey enfumé en Afrique de l'Ouest renforça cette hypothèse. De plus, les similitudes d'organisation de leur génome (présence du gene vpx), la superposition géographique entre l'épicentre de l'épidémie du VIH-2 et l'aire de répartition des mangabeys enfumés, et enfin le fait que ces singes sont régulièrement chassés ou gardés comme animaux domestiques, ont permis d'identifier le SIVsmm comme étant l'origine du VIH-2.

\section{Origine du VIH-1}

Les premières souches de SIVcpz furent isolées, il y a 15 ans, chez deux chimpanzés captifs mais nés dans la nature au Gabon, SIVcpz, GABl et GAB2. L'analyse

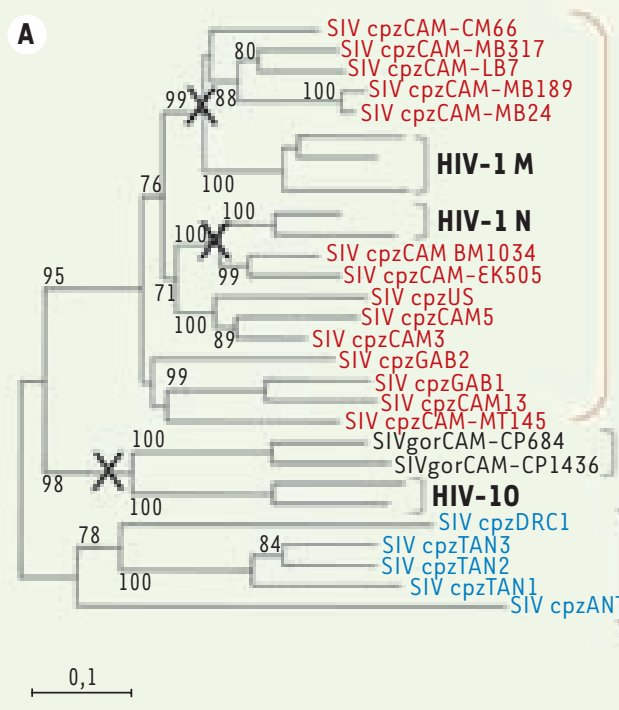

B

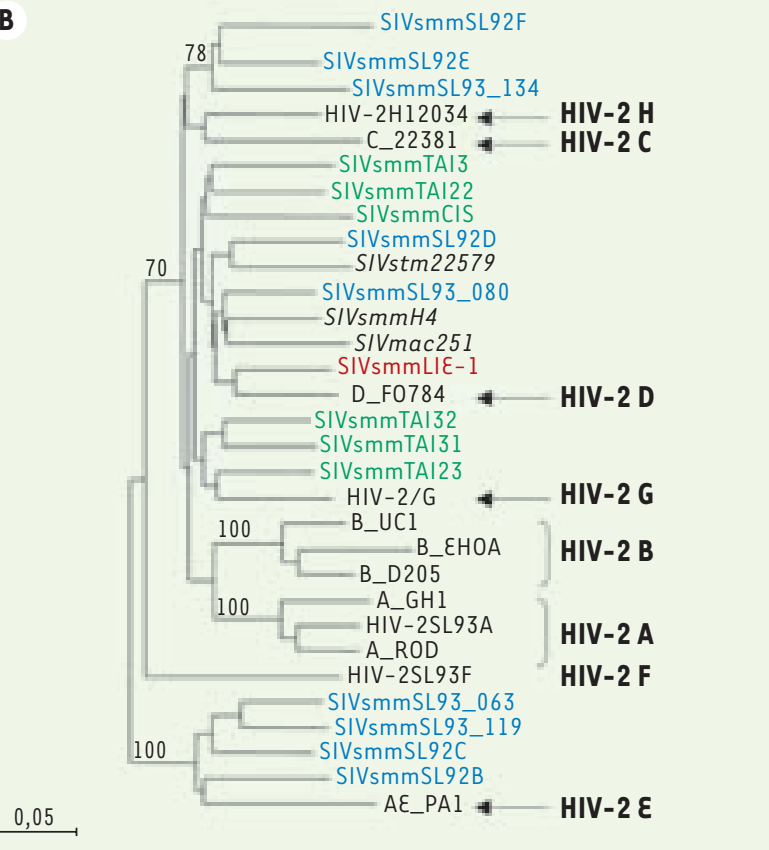

SIVcpzPts
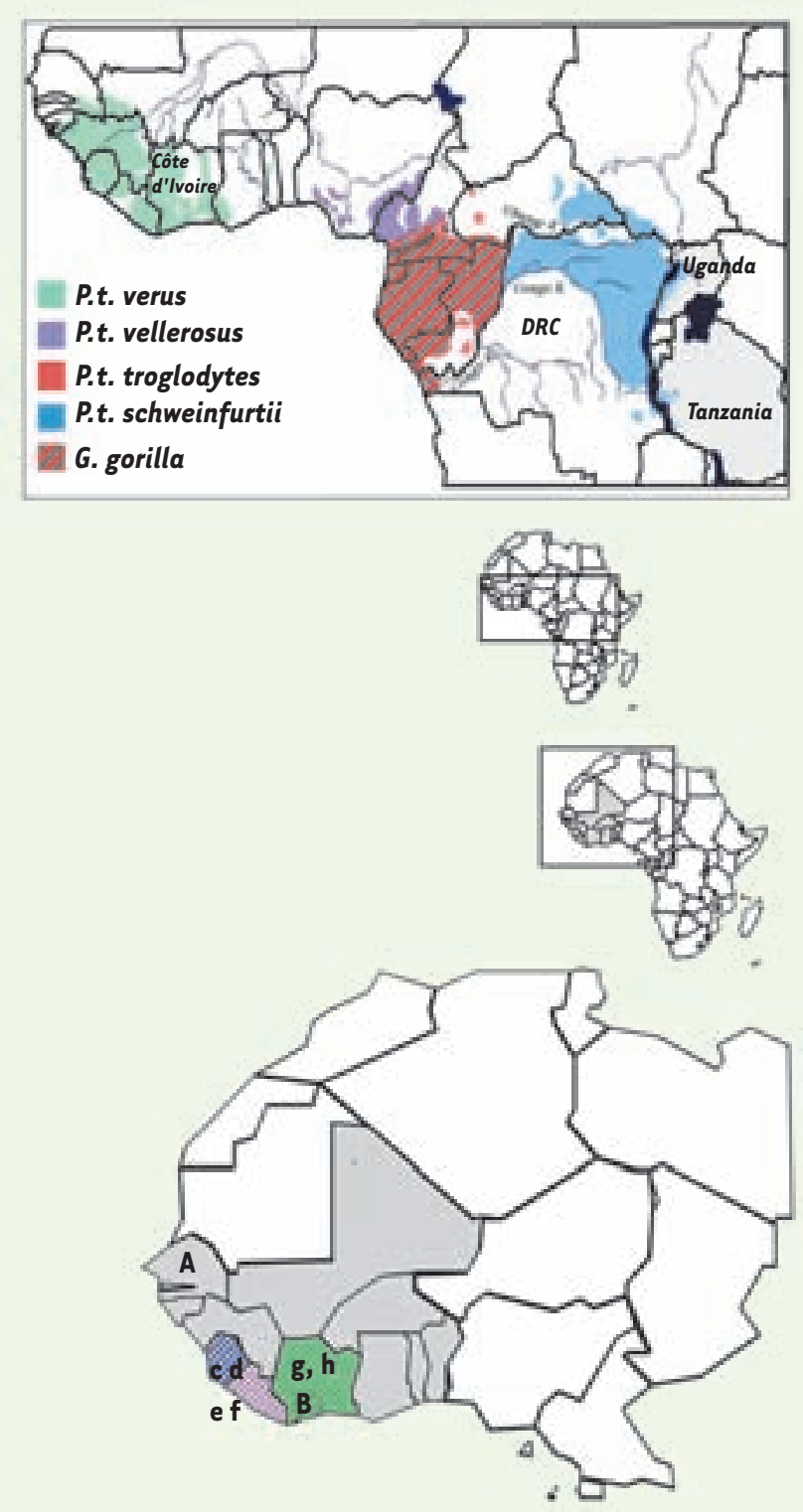

Figure 3. A. Habitat des singes. La carte géographique représente l'habitat des différentes sous-espèces de chimpanzés ainsi que des gorilles. L'arbre phylogénétique illustre la proximité phylogénétique des séquences de VIH-l groupe $M$ et $\mathrm{N}$ avec les séquences de SIVcpzPtt ainsi que la proximité des séquences de SIVgor avec les séquences de VIH-l groupe 0 . Les $X$ représentent les 3 transmissions inter-espèces de virus SIV à I'homme. B. La carte géographique représente l'habitat des singes mangabey enfumé (Cercocebus atys). L'arbre phylogénétique illustre la proximité phylogénétique des différents groupes de VIH-2 avec les séquences de SIVsmm. 
du génome du SIVcpzGabl révéla la présence du gène accessoire $v p u$, également présent dans le VIH-l. De plus, l'analyse phylogénétique de ce virus a montré qu'il était apparenté au VIH-l et non pas aux autres SIV connus à l'époque. L'étude de chimpanzés captifs permit la caractérisation partielle ou complète de plusieurs souches SIVcpz, trois SIVcpzCAM furent isolés chez des chimpanzés au Cameroun, SIVcpzUS le fût d'un chimpanzé vivant dans un zoo américain et SIVcpzANT d'un chimpanzé importé illégalement en Belgique et provenant de la République Démocratique du Congo (RDC). Toutes ces souches ont été isolées chez des chimpanzés de la sous-espèce $P$. t. troglodytes. Exceptée SIVcpzANT, isolée chez un chimpanzé $P$. $t$. schweinfurthii, qui se montra très divergente des autres souches SIVcpz. Cette observation suggèra qu'il existait deux lignées SIVcpz spécifiques de leur sous-espèce d'origine. Toutes les souches VIH-l s'avéraient plus proches du SIVcpzPtt que du SIVcpzPts.

Si ces données suggéraient fortement que les chimpanzés d'Afrique Centrale de l'Ouest étaient la source du virus VIH-1, la mise en évidence du réservoir de façon définitive imposait de pouvoir étudier un nombre plus important de chimpanzés vivant dans la nature [16]. La difficulté majeure d'une telle étude repose sur le fait qu'il s'agit d'une espèce protégée et menacée de disparition. Il est donc important de ne pas perturber ces animaux dans leur environnement naturel que sont les zones de forêt les plus reculées. La mise au point d'une technique non invasive, permettant la détection et la caractérisation de SIVcpz dans les fèces, était donc un préalable pour permettre la réalisation de véritables études d'épidémiologie moléculaire en Afrique Centrale. Par cette méthode, de nouvelles souches de SIVcpz provenant de chimpanzés vivant en Tanzanie et en RDC et appartenant à la sous-espèce $P$. $t$. schweinfurthii ont été caractérisées. Toutes ces nouvelles souches SIVcpzPts forment une lignée bien séparée, proche du SIVcpzANT, confirmant que cette lignée n'est pas à l'origine du VIH-1 [19, 20]. En Afrique Centrale de l'ouest, une étude récente dans différentes communautés de chimpanzés du Sud Cameroun a montré une prévalence du SIVcpz variant de 4 à $35 \%$ en fonction des groupes. Plus de trente nouvelles souches de SIVcpz furent identifiées, proches des autres souches de SIVcpzPtt. De plus, pour la première fois, des souches SIVcpz étaient identifiées sur la totalité de leurs gènes, très proches des $\mathrm{VIH}-1$ du groupe $M$ et $\mathrm{du}$ groupe N (Figure 3A). En revanche, aucune n'était proche du groupe 0 . Les nouveaux SIVcpzPtt montraient des groupements géographiques de virus permettant ainsi de mieux définir les zones géographiques « considérées comme berceaux de l'épidémie de VIH-1»[1, 17].
Néanmoins, des régions frontalières moins étudiées à ce jour pourraient également être considérées comme «berceaux » de l'épidémie.

\section{Origine du VIH-1 groupe 0 :}

\section{l'infection SIV du gorille?}

Récemment, un virus SIV a été décrit dans une seconde espèce de grand singe au Cameroun, le gorille de l'Ouest (Gorilla gorilla gorilla). De façon surprenante, tous les nouveaux virus provenant de gorilles, appelés SIVgor, formaient un groupe apparenté au VIH-1 du groupe 0. Cependant, les relations phylogénétiques entre SIVcpz, SIVgor et VIH- I indiquent que les chimpanzés représentent le réservoir original des SIV maintenant retrouvés chez les chimpanzés, les gorilles et l'homme. Les gorilles étant herbivores et n'ayant apparemment pas de contacts physiques avec d'autres primates et en particulier avec les chimpanzés, la façon dont les gorilles se sont contaminés reste un mystère [2]. II n'est pas encore clairement établi si des chimpanzés ont été à l'origine d'une double transmission - d'une part au gorille et responsable du SIVgor, et d'autre part à l'homme et responsable du VIH-l groupe 0 - ou si seul le gorille a pu transmettre l'ancêtre du VIH-1 groupe 0 à l'homme. Cependant, il sera important d'étudier plus en détail les infections SIV chez les gorilles et chez les chimpanzés sauvages pour confirmer cette hypothèse et vérifier si tous les SIVgor sont proches du VIH-1 groupe 0 . Cela permettra aussi d'identifier d'éventuels réservoirs SIVgor-like chez les chimpanzés. Nos connaissances actuelles se fondent surtout sur des données issues des grands singes du Cameroun et il est aussi important d'aller les étudier dans d'autres zones géographiques telles que le Gabon, le Congo, la République Centrafricaine ou la Guinée Équatoriale.

\section{Transmission inter-espèces du singe à l'homme : où, quand et comment les transmissions inter-espèces du singe à l'homme se sont-elles produites?}

Dans la mesure où les groupes de VIH-l retrouvés chez l'Homme s'intègrent dans la même radiation phylogénétique, VIH-1/SIVcpz/SIVgor, la transmission inter-espèces a eu lieu le plus probablement en Afrique Équatoriale de l'Ouest, aire de répartition des gorilles de l'Ouest et du chimpanzé $P$. $t$. troglodytes. Ainsi l'origine des trois groupes de virus VIH-l est clairement l'Afrique Centrale de l'ouest. Mais alors que les virus du groupe M ont connu une diffusion mondiale, le VIH-l du groupe 0 est resté localisé au Cameroun et aux pays limitrophes (Gabon, Nigéria, Guinée Equatoriale). Quant au VIH-l du groupe N, il n'a été retrouvé que chez quelques personnes vivant au Cameroun [21]. Si le virus ancestral du VIH-1 M a bien été identifié au Cameroun, il n'en demeure pas moins que la plus grande diversité génétique du VIH-I M en termes de nombre de sous-types et de diversité intra soustype a été observée dans la partie occidentale de la RDC suggérant que l'épicentre de l'épidémie du VIH-1 du groupe M est situé dans cette région d'Afrique $[22,23]$. Les études d'horloge moléculaire estiment la date de l'ancêtre commun de tous les VIH-1 M à 1930 [IC : 1915-1941] [24, 25]. Une période similaire est également estimée pour l'origine du VIH-1 du groupe $0: 1920$ [IC: 1891-1940] [26]. 
Une relation phylogénétique étroite est également observée entre le SIVsmm du mangabey enfumé (Cercocebus atys) et le virus VIH-2 d'Afrique de l'Ouest. L'aire de répartition de ces singes s'étend du Sénégal à la Côte d'Ivoire et se superpose à l'épicentre de l'émergence du VIH-2. Huit groupes de VIH-2 (A-H) ont été décrits à ce jour, mais seuls les groupes $A$ et $B$ ont connu une diffusion épidémique, le soustype $A$ circulant principalement en Guinée Bissau et au Sénégal tandis que le sous-type B est retrouvé en Côte d'Ivoire [27-29]. La plupart des autres groupes ( $\mathrm{C}-\mathrm{H})$ n'infectent que peu d'individus (Figure $3 B$ ). Les études d'horloge moléculaire font remonter l'émergence du VIH-2 aux années 1940 pour le groupe $A$ (avec un intervalle de confiance de 16 ans) et 1945 pour le groupe $B$ (avec un intervalle de confiance de 14 ans) [30].

Bien que le mode exact de transmission des virus simiens (SIVcpz et SIVsmm) à I'homme ne soit pas connu, l'exposition à du sang ou à des sécrétions d'animaux infectés à l'occasion de la chasse ou de la préparation de la viande de brousse semble la cause la plus probable de contamination. Les morsures de singes captifs peuvent également avoir été un autre mode de contamination. Ces facteurs à l'origine de l'émergence du virus chez l'homme sont bien sûr à différencier de ceux qui ont favorisé sa diffusion épidémique, ceux-ci étant multifactoriels (comportementaux, environnementaux...).

\section{Conclusion}

Il est vrai qu'il existe maintenant une bonne connaissance sur l'origine du VIH-1, mais les facteurs impliqués dans la pandémie de VIH/Sida qui s'est développée par la suite ne sont pas clairement définis. Le SIVcpzPtt, à l'origine du VIH-l des groupes $M$ et $\mathrm{N}$ est toujours présent dans les populations de chimpanzés Pan troglodytes troglodytes du Sud Cameroun. Le VIH-1 du groupe 0 est présent chez le gorille de l'Ouest (Gorilla gorilla) mais les chimpanzés sont le réservoir original du SIVgor et il reste à établir si le VIH-1 du groupe 0 a été transmis à l'Homme par le gorille et/ou par le chimpanzé. Le SIVsmm est, lui, à l'origine du VIH-2 et au moins huit transmissions inter-espèces de SIVsmm ont eu lieu entre I'homme et le mangabey enfumé, correspondant aux 8 groupes HIV-2. L'épidémie actuelle de VIH démontre l'extraordinaire importance que peut avoir un seul épisode de transmission de lentivirus. Dans la mesure où l'homme est toujours potentiellement exposé à de nombreux SIV du fait de la chasse ou de la préparation de viande de brousse, la possibilité de nouveaux épisodes de transmissions inter-espèces de lentivirus de primates est une éventualité qu'il faut anticiper. De nombreux éléments sont néanmoins indispensables pour qu'une simple transmission virale soit à l'origine d'une nouvelle épidémie. Une première étape après le passage de la barrière d'espèce est l'adaptation de ce virus à son nouvel hôte, indispensable pour que le virus diffuse à travers la population. Les facteurs de l'hôte, associés aux facteurs environnementaux, sociaux, démographiques, sont les principaux à jouer un rôle pour la diffusion d'un tel nouveau virus [31]. $\diamond$

\section{SUMMARY}

\section{Genetic diversity}

and phylogeographic distribution of SIV :

how to understand the origin of HIV

Emergence of human immunodeficiency viruses HIV-1 and HIV-2 results from interspecies transmission from simian viruses SIV. SIVcpzPtt infecting chimpanzees, and from which the HIV-1 (subgroups $M$ and N) is derived is still found in the Pan troglodytes troglodytes population of south Cameroon chimpanzees. The ancestor of HIV-1 group 0, is found in the Gorilla residing in Western Africa, but chimpanzees are in fact the initial reservoir of the SIV viruses SIVgor, and it is still unclear whether the group 0 HIV-1 has been trasmitted to humans by gorillas and/or chimpanzees. At least eight interspecies transmissions between and humans implicating SIVsmm (from sooty mangabey monkeys) have occured, corresponding to the eight VIH-2 groups. Since habits of hunting and meat preparation in the bush still persistently expose humans in Africa to SIV infection, new interspecies transmission of these viruses remains a possibility. $\diamond$

\section{À RETENIR}

- La phylogénie des lentivirus de primates (non-humains) est extrêmement complexe et caracterisée par co-évolution, recombinaison, surinfection et transmissions inter-espèces.

- Le SIVsmm du mangabey enfumé (Cercocebus atys) en Afrique de l'Ouest est identifié comme l'origine du VIH-2.

- Le SIVcpzPtt est à l'origine du VIH-1, groupes M et N, et est toujours présent dans les populations des chimpanzés de l'Afrique Centrale de l'ouest (Pan troglodytes troglodytes) du Sud Cameroun.

- Le gorille de l'Ouest (Gorilla gorilla gorilla) est infecté par un virus, SIVgor, proche du VIH-1 groupe 0.

- Les facteurs à l'origine de l'émergence du virus VIH chez l'Homme sont à différencier de ceux qui ont favorisé sa diffusion épidémique. 


\section{RÉFÉRENCES}

1. Keele BF, Van Heuverswyn F, Li Y, et al. Chimpanzee reservoirs of pandemic and nonpandemic HIV-1. Science 2006 ; $313: 523-6$

2. Van Heuverswyn F, Li Y, Neel C, et al. Human immunodeficiency viruses : SIV infection in wild gorillas. Nature $2006 ; 444: 164$.

3. Santiago ML, Range F, Keele BF, et al. Simian immunodeficiency virus infection in free-ranging sooty mangabeys (Cercocebus atys atys) from the Tai Forest, Cote d'Ivoire: implications fo the origin of epidemic human immunodeficiency virus type 2. J Virol $2005 ; 79: 12515-27$.

4. VandeWoude S, Apetrei C. Going wild : lessons from naturally occurring T-lymphotropic lentiviruses. Clin Microbiol Rev 2006 ; 19 : 728-62.

5. Aghokeng AF, Peeters M. Simian immunodeficiency viruses (SIVs) in Africa. J Neurovirol 2005 ; 11 (suppl 1) : 27-32.

6. Apetrei C, Marx PA. African lentiviruses related to HIV. J Neurovirol 2005 ; $11: 33-49$.

7. Bibollet-Ruche F, Bailes $E$, Gao F, et al. New simian immunodeficiency virus infecting De Brazza's monkeys (Cercopithecus neglectus) : evidence for a cercopithecus monkey virus clade. J Virol $2004 ; 78: 7748-62$.

8. Vidal N, Mulanga C, Bazepeo SE, et al. Identification and molecular characterization of subsubtype A4 in central Africa. AIDS Res Hum Retrovir 2006; 22 : 182-7.

9. Geretti AM. HIV-1 subtypes : epidemiology and significance for HIV management. Curr Opin Infect Dis $2006 ; 19: 1-7$.

10. Chaix ML, Descamps D, Harzic M, et al. Stable prevalence of genotypic drug resistance mutations but increase in non-B virus among patients with primary HIV-l infection in France. Aids $2003 ; 17: 2635-43$.

11. Chaix ML, Deveau C, Goujard C, et al. Increase of the HIV-1 non-B subtypes frequency and response to HAART in patients enrolled in the French Primo cohort study and treated at the time of primary infection. Denver: $13^{\text {th }}$ Conference on Retroviruses and Opportunistic Infections, 2006.

12. Descamps D, Chaix ML, Andre P, et al. French national sentinel survey of antiretroviral drug resistance in patients with HIV-1 primary infection and in antiretroviral-naive chronically infected patients in 2001-2002. J Acquir Immune Defic Syndr 2005 ; 38: 545-52.

13. Semaille C, Barin F, Cazein F, et al. Monitoring the dynamics of the HIV epidemic using assays for recent infection and serotyping among new HIV diagnoses : experience after 2 years in France. J Infect Dis 2007 ; $196: 377-83$.

14. Bailes $\varepsilon$, Gao F, Bibollet-Ruche F, et al. Hybrid origin of SIV in chimpanzees. Science 2003; $300: 1713$

15. Switzer WM, Parekh B, Shanmugam V, et al. The epidemiology of simian immunodeficiency virus infection in a large number of wild-and captive-born chimpanzees : evidence for a recent introduction following chimpanzee divergence. AIDS Res Hum Retrovir 2005 ; $21: 335-42$

16. Sharp PM, Shaw GM, Hahn BH. Simian immunodeficiency virus infection of chimpanzees. J Virol $2005 ; 79: 3891-902$.

17. Van Heuverswyn F, Li Y, Bailes $\varepsilon$, et al. Genetic diversity and phylogeographic clustering of SIVcpzPtt in wild chimpanzees in Cameroon. Virology 2007 ; $368: 155-71$.

18. Apetrei C, Kaur A, Lerche NW, et al. Molecular epidemiology of simian immunodeficiency virus SIVsm in US primate centers unravels the origin of SIVmac and SIVstm. J Virol 2005 ;

$79: 8991-9005$
19. Santiago ML, Lukasik M, Kamenya S, et al. Foci of endemic simian immunodeficiency virus infection in wild-living eastern chimpanzees (Pan troglodytes schweinfurthii). J Virol 2003; 77 : 7545-62.

20. Worobey M, Santiago ML, Keele BF, et al. Origin of Aids : contaminated polio vaccine theory refuted. Nature $2004 ; 428: 820$.

21. Ayouba A, Souquières $S$, Njinku B, et al. HIV-1 group N among HIV-1 seropositive individuals in Cameroon. Aids $2000 ; 14: 2623-5$.

22. Vidal N, Mulanga C, Bazepeo SE, et al. Distribution of HIV-1 variants in the Democratic Republic of Congo suggests increase of subtype $C$ in Kinshasa between 1997 and 2002. J Acquir Immune Defic Syndr 2005; $40: 456-62$.

23. Kalish ML, Robbins KE, Pieniazek D, et al. Recombinant viruses and early global HIV-1 epidemic. Emerg Infect Dis 2004 ; 10 : 1227-34.

24. Korber B, Muldoon M, Theiler J, et al. Timing the ancestor of the HIV-1 pandemic strains. Science $2000 ; 288: 1789-96$.

25. Salemi M, Strimmer K, Hall WW, et al. Dating the common ancestor of SIVcpz and HIV-1 group M and the origin of HIV-l subtypes using a new method to uncover clock-like molecular evolution. Faseb J 2001; $15: 276-8$.

26. Lemey $P$, Pybus $O G$, Rambaut $A$, et al. The molecular population genetics of HIV-1 group 0 . Genetics $2004 ; 167$ : 1059-68.

27. Pieniazek D, Rayfield M, Hu DJ, et al. HIV-2 protease sequences of subtypes $A$ and $B$ harbor multiple mutations associated with protease inhibitor resistance in HIV-1. Aids $2004 ; 18:$ 495-502.

28. Plantier JC, Gueudin M, de Oliveira F, et al. Rapid discrimination between human immunodeficiency virus type 2 groups $A$ and $B$ by real-time PCR. J Clin Microbiol $2004 ; 42$ : 5866-70.

29. Damond F, Worobey M, Campa P, et al. Identification of a highly divergent HIV type 2 and proposal for a change in HIV type 2 classification. AIDS Res Hum Retrovir $2004 ; 20: 666-72$

30. Lemey P, Pybus OG, Wang B, et al. Tracing the origin and history of the HIV-2 epidemic. Proc Natl Acad Sci USA 2003 ; 100 : 6588-92.

31. Heeney JL, Dalgleish AG, Weiss RA. Origins of HIV and the evolution of resistance to AIDS. Science 2006 ; 313: 462-66.

32. Anglaret X, Salamon R. Épidémie de sida en Afrique subsaharienne. Med Sci (Paris) $2004 ; 20: 593-8$.

33. Darlix JL, Lévy Y. Le virus du Sida au milieu du gué vingt-cinq ans après. Med Sci (Paris) $2008 ; 24: 4-6$.

34. Courgnaud V, Müller-Trutwin M, Sonigo P. Évolution et virulence des lentivirus de primates. Med Sci (Paris) $2004 ; 20: 448-52$.

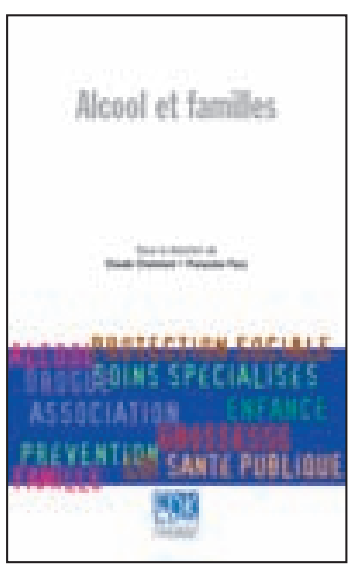

ISBN : 978-2-8425-4116-3 174 pages

\section{Bon de commande}

À retourner à EDK, 2, rue Troyon - 92316 Sèvres Cedex

Tél. : 0155641393 - Fax : 0155641394 - E-mail : edk@edk.fr

NOM : Prénom :

Adresse :

Code postal : Ville :

Pays :

Fonction :

Je souhaite recevoir l'ouvrage Alcool et familles : $15 €+3 €$ de port $=\mathbf{1 8} €$ TTC

en ................. exemplaire, soit un total de .......................................... €

$\square$ Par chèque, à l'ordre de $\mathbf{E} \mathbf{D}$ K

$\square$ Par carte bancaire :

$\square$ Visa $\square$ Eurocard/Mastercard

Carte $n^{\circ} 1 \quad 1 \quad 1 \quad 1 \quad 1 \quad 1 \quad 1 \quad 1 \quad 1 \quad 1 \quad 1 \quad 1 \quad 1 \quad 1 \quad 1 \quad 1 \quad 1 \quad 1 \quad 1 \quad 1$

Date d'expiration: $\quad \leq 1 \mid$

$\mathrm{N}^{\circ}$ de contrôle au dos de la carte :
TIRÉS À PART

M.L. Chaix 